\title{
Recent Prescribing Patterns of Cardiac Physicians in Metropolitan Dhaka City, Bangladesh
}

\author{
Kazi Ruhullah Shahriar ${ }^{1}$ and Muhammad Rashedul Islam² \\ ${ }^{1}$ Department of Pharmaceutical Chemistry, Faculty of Pharmacy, University of Dhaka \\ Dhaka-1000, Bangladesh \\ ${ }^{2}$ Department of Pharmaceutical Technology, Faculty of Pharmacy, University of Dhaka \\ Dhaka-1000, Bangladesh
}

(Received: 18 March, 2018; Accepted: 31 May, 2018; Published: 31 July, 2018)

\begin{abstract}
A large number of people in Bangladesh suffer from many cardiovascular diseases (CVDs). Therefore, cardiovascular drugs are one of the major classes of drugs prescribed in Bangladesh. The study of prescribing patterns seeks to monitor, evaluate and suggest modifications in clinicians' prescribing habits to make medical care more rational. This study was mainly focused on the recent trends of prescribing patterns of oral cardiovascular drugs in Dhaka city. It was a questionnaire-based direct interview type of descriptive study, conducted in the cardiology departments of eight most renowned hospitals of Dhaka, during the study period of February 2017 to April 2017. Most patients in the disease states belonged to the older age group of 50-60 years. More male patients were found in the cardiology departments than the female patients. Hypertension was the most prevalent diseases $(86.67 \%)$ followed by angina $(75.00 \%)$ and myocardial infarction $(53.33 \%)$. Most commonly prescribed antihypertensive medication was angiotensin-II receptor blocker (ARB, 55.00\%) and individually losartan (61.67\%), followed by angiotensin-converting enzyme inhibitor (ACEI, 25.00\%) and only ramipril (90.00\%). Amlodipine was the first choice of every respondent when prescribing a calcium-channel blocker (CCB). In case of combination products, amlodipine plus atenolol was found to be the most preferred $(65 \%)$ medication, followed by hydrochlorothiazide plus losartan (40\%).
\end{abstract}

Key words: Cardiovascular diseases, prescribing patterns, cardiovascular drugs, drug combinations.

\section{Introduction}

Research studies of prescription behavior of physicians is increasing globally and it is being carried out in different health care setups for observing the rational use of drugs in the society. It is a powerful exploratory tool to assess the present patterns of drug use and appropriateness of prescriptions. This kind of studies has massive socioeconomic and medical significant value and is needed to identify the trends as well as to set the priorities. This study conducted not only in the interest of the regulatory control, but also as a basis of national public health issues (Dukes, 1993; Pendhari et al., 2013). most frequent cause of morbidity and mortality throughout the world (Andrew and Eugene, 2006).The Global Burden of Disease (GBD) study estimated that $29.6 \%$ of all deaths worldwide (15616.1 million deaths) were caused by cardiovascular diseases (CVD's) in 2010, more than all communicable, maternal, neonatal and nutritional disorders combined, and double the number of deaths caused by cancers (Lozano et al., 2012; Kumar et al., 2016).It was reported that by 2030, almost 25 million people would die from CVDs, mainly from heart disease and stroke (Manjula et al., 2012; Labu et al., 2013).

Correspondence to: Muhammad Rashedul Islam; Tel: +88-01718848178, Fax: + 88-02-9667222; E-mail: rashed1505@yahoo.com 
Important modifiable risk factors of CVDs are unhealthy diet, physical inactivity, tobacco use and the effects insinuate abnormal blood lipid profile and obesity. Less physical activity and excessive fat rich diet are two major health concerns in affluent society. A study in Bangladesh revealed that 27.93, 21.08 and $13.41 \%$ stroke patients with lipid disorder had high cholesterol, low-density lipoprotein (LDL) and triglycerides (TG) level, respectively. However, the study also showed that $42.67 \%$ patients had low high density lipoprotein (HDL) level showed in the same study (Comeau et al., 1998; Badiuzzamanet al., 2009; Muhit et al., 2012).

There are numerous classes of cardiovascular drugs available worldwide. Diuretics, angiotensinconverting enzyme inhibitors (ACEIs), angiotensin-II receptor blockers (ARBs), calcium-channel blockers (CCBs), beta-adrenoceptor blockers (BBs), etc. are the major categories. To study the prescribing trends of cardiovascular drugs in Dhaka, some prescriptionbased surveys were done before (Hossain et al., 2015). However, in this study, we decided not only to meet the target physicians of Dhaka randomly; rather we selected the most renowned hospitals in this disease treatment section.

Is the pharmaceutical companies have made most of the cardiovascular drugs recognized worldwide available in Bangladesh, the physicians have a variety of preferences when prescribing these drugs. Therefore, from a clinical as well as marketing perspective, there is a need to study which cardiovascular drugs are more popular among the physicians of Bangladesh. For this reason, this study was designed to get an idea of the preference of the cardiac physicians of the metropoliton Dhaka city.

\section{Research Methods}

This study was mainly a questionnaire-based interview. It was conducted using a semi-structured questionnaire containing multiple-choice questions. In this method, a form containing a predetermined set of questions was used (Gill et al., 2008). The data was collected after obtaining consent from the participating physicians and on the scheduled appointment time. They were informed all the ethical issues and given assurance of confidentiality of their data. Relevant information was gathered from reliable books and articles from peer-reviewed journal articles to prepare the questionnaire for this survey. Journal articles that provided information with respect to Bangladesh were prioritized.

Physicians from Cardiology Department of Dhaka Medical College Hospital (DMCH), National Institute of Cardiovascular Diseases (NICVD), Apollo Hospitals Dhaka (AHD), Bangabandhu Sheikh Mujib Medical University (BSMMU), National Heart Foundation Hospital \& Research Institute (NHFH \& RI), United Hospital Limited (UHL), Ibrahim Cardiac Hospital and Research Institute, and Islami Bank Central Hospital were included in this study. The study was carried out between the period of February 16, 2017 and April 16, 2017. The sample size was limited to 60 .

The sampling method was purposive sampling, where the individuals of a population who are especially knowledgeable and experienced were deliberately selected (Palinkas et al., 2015). Such sampling was useful in this regard because focusing on prominent physicians of the country were supposed to give us a better and non-biased picture of the prescription pattern. The data collected from the survey was tabulated, analyzed and graphically presented using Microsoft Office Excel 2016.

\section{Results and Discussion}

Number of respondents from various hospitals: Total 60 (sixty) physicians from 08 (eight) most renowned hospitals of Dhaka city were interviewed and their responses were recorded for this survey. A total of 17 respondents were from $\mathrm{DMCH}, 7$ were from BSMMU and 13 were from NICVD - these public hospitals provide treatment of high quality with minimum possible cost. On the other hand, 9 respondents were from AHD and 5 were from UHL, which are tertiary private hospitals with modern facilities and high cost. Besides those, 5 respondents NHFH \& RI and 4 were from Ibrahim Cardiac 
Hospital and Research Institute, which are specialized hospitals for cardiac patients.

Location of hospitals and physicians' profile: The selected location of those hospitals in Dhaka city is shown in Figure 1. It shows that different areas of Dhaka city were covered. In addition, all the respondents had or wash or having either MD in
Cardiology or D. Card. Degree and were working in cardiology department of those hospitals as professor, associate professor, assistant professor, consultant, registrar and other posts. Therefore, we can say that the survey was strong enough to get an overall picture about the prescribing trends of cardiovascular drugs in Dhaka city.

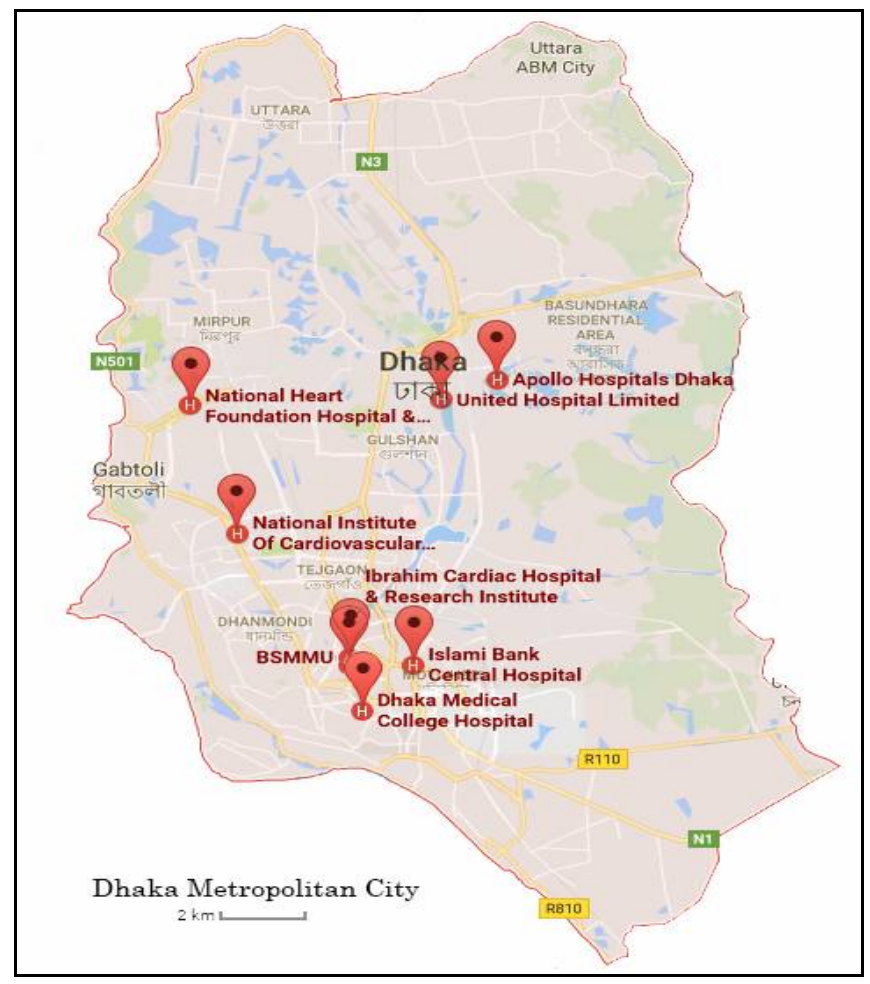

Figure 1. Location of different hospitals in Dhaka city where the study was conducted.

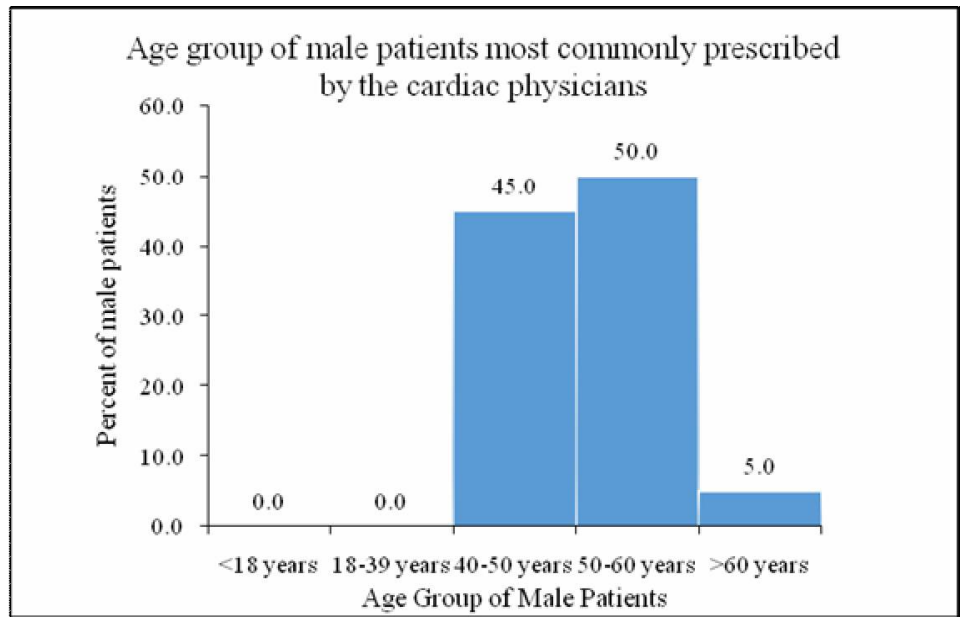

Figure 2.Age group of male patients receving treatments from the cardiac physicians. 
Common group of cardiac patients: $95 \%$ of the respondents agreed that male patients were more prone to have cardiovascular diseases. $50 \%$ respondents answered that males and females aged between 50 to 60 years were in significant risk (Figure 2).

Common cardiovascular diseases: The physicians were asked about the types of cardiovascular diseases that are commonly encountered by them. Most of the respondents gave multiple answers to this question because majority of the cardiac patients are prone to have multiple diseases concurrently. Hypertension got the highest response $(86.67 \%)$ followed by angina $(75 \%)$ and myocardial infarction (MI, 53.33\%). Other diseases like coronary heart failure (CHF, 18.33\%), arrhythmia (11.67\%), cardiomyopathy $(8.33 \%)$ and vascular heart disease (VHD, 6.67\%) got lower responses (Figure 3).

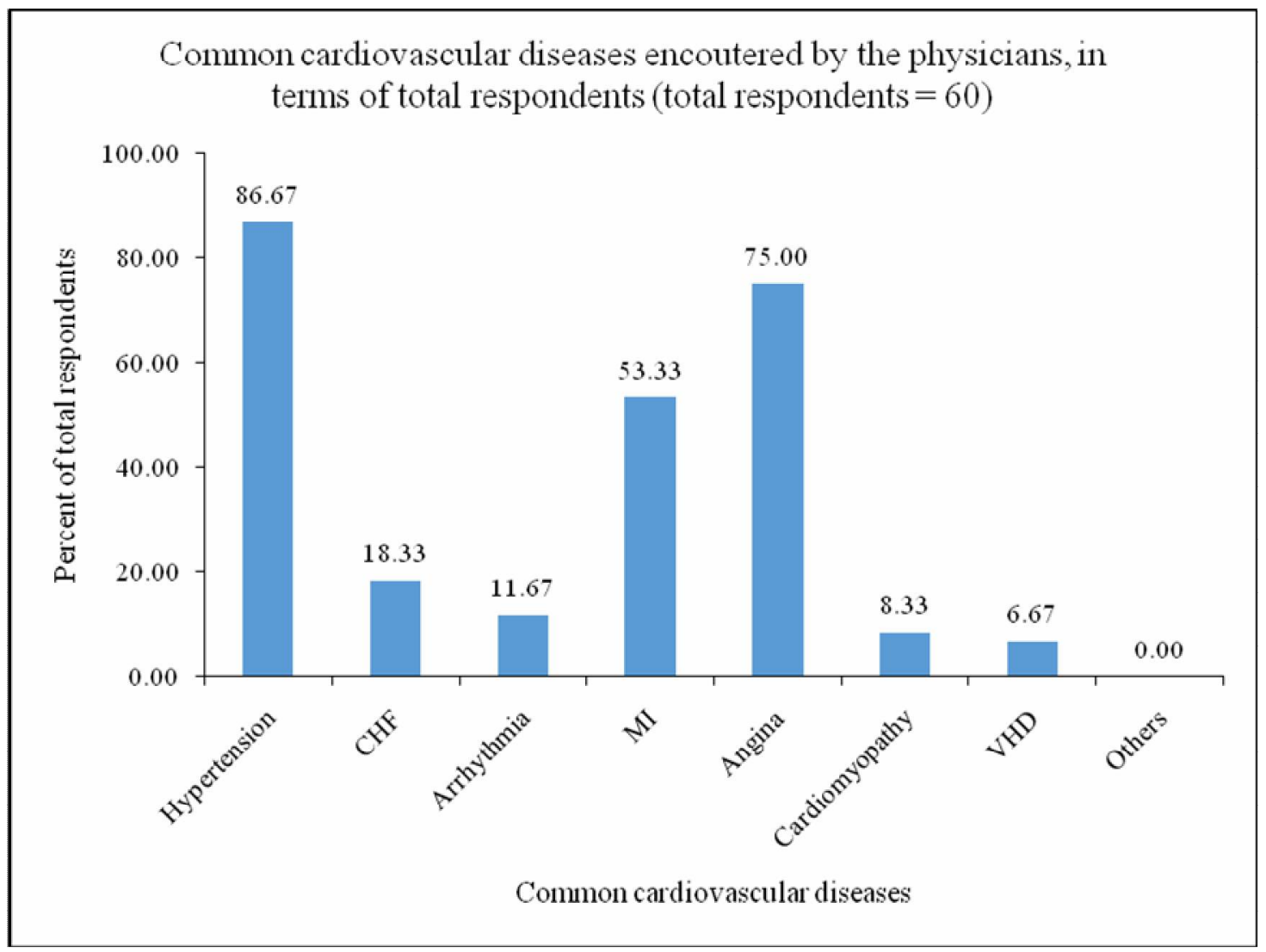

Figure 3. Common cardiovascular diseases encountered by the physicians.

Preferred class of antihypertensive drugs: With the cardiovascular market, antihypertensives represent the largest class that share $22 \%$ of the overall market (Business Insights, 2011). Many participants responded with multiple answers when they were asked about the drug class they usually prefer as the first choice in the treatment of hypertension. Figure 4 shows that, ARBs got the highest response (55\%) as the most preferred class for the treatment of hypertension. 25\% respondents preferred ACEIs whereas $18.33 \%$ picked CCBs. Thiazide (3.33\%) and BBs (1.67\%) got lower responses because they are not considered as good first line choice as monotherapy. However, 25\% 
respondents upgraded to combination rather than monotherapy. ARB plus CCB combination got the maximum response (13.33\%). In addition to this, combinations of CCB plus BB and ARB plus thiazide got $5 \%$ response and ACEI plus thiazide combination was preferred by $1.67 \%$ of total respondents (Table 1). The popularity of ARBs has been primarily due to their favorable side-effect profile, which has given them a competitive edge over several ACE inhibitors. This class of antihypertensive were increasingly prescribed worldwide and considered as one of the growth drivers in this global market (Business Insights, 2011).

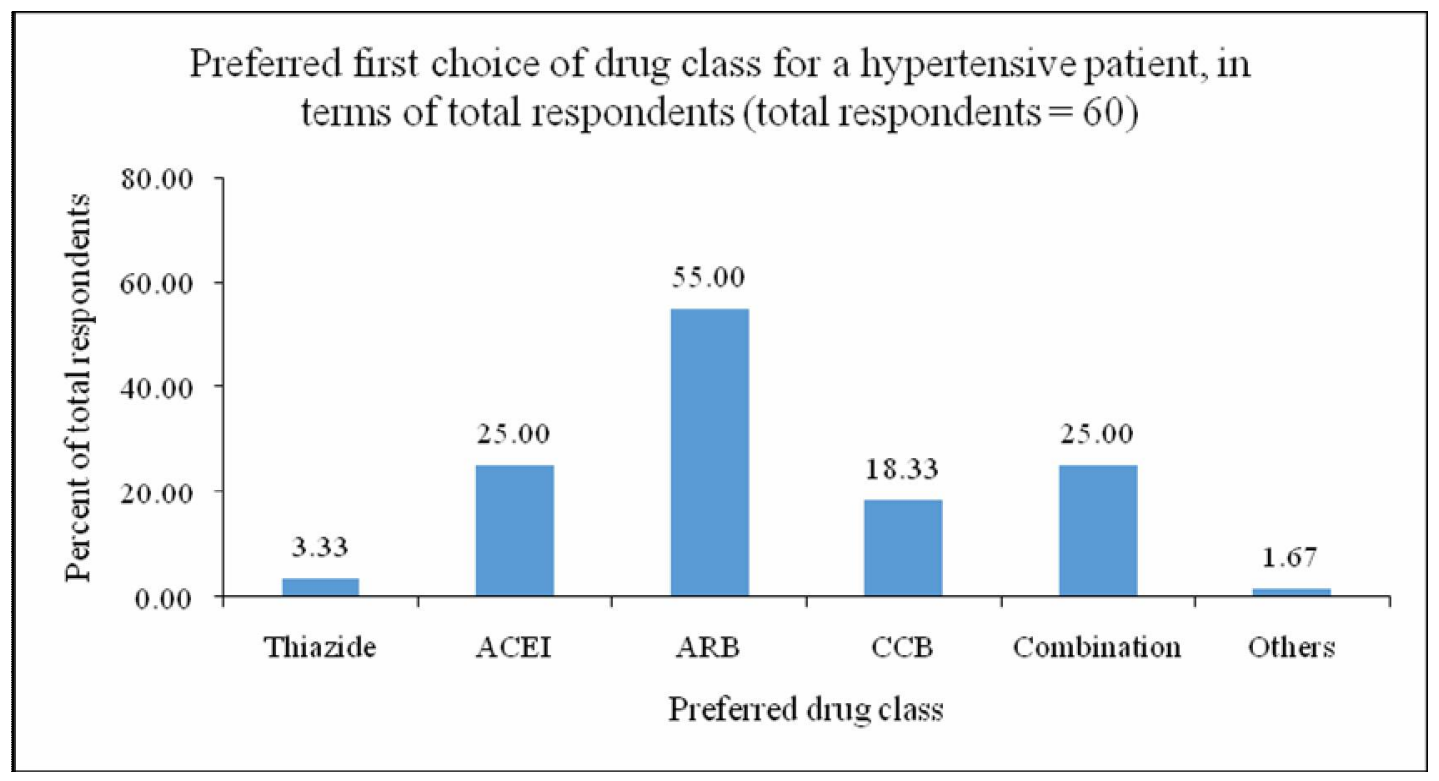

Figure 4. Preferred first choice of drug class for hypertensive patients.

Table 1. Combination choice for hypertensive patients.

\begin{tabular}{lll}
\hline Combinations & No. of Responses & Percentage of Total Respondents \\
\hline ARB plus CCB & 8 & 13.33 \\
CCB plus BB & 3 & 5.00 \\
ARB plus Thiazide & 3 & 5.00 \\
ACEI plus Thiazide & 1 & 1.67 \\
\hline
\end{tabular}

Preferred ACEI: Ramipril was found to be the most popular (90\%) among the ACEIs. perindopril (6.67\%), captopril (3.33\%) and enalapril (1.67\%) got lower responses. However, fosinopril and lisinopril got no response (Table 2). The reason why ramipril is so popular is probably due to the fact that, studies have proven ramipril being particularly effective in treating people who have diabetes and other heart related risk factors, and in the prevention of kidney function decline in diabetics (Doggrell, 2001).

Preferred ARB: Angiotensin-II receptor antagonists are known to have good efficacy and side-effect profiles (Business Insights, 2011). Among the ARBs, losartan was the most prescribed (61.67\%) one. Olmesartan (33.33\%) and valsartan (21.67\%) came next. Only $1.67 \%$ of total respondents cited irbesartan. Telmisartan and candesartan got no 
response (Figure 5). Previous studies showed that losartan was the only ARB to significantly lower uric acid levels (Wolff et al., 2015). This is probably the reason why losartan is so popular. On the other hand, olmesartan gets preference due to the fact that, it is more effective than valsartan and losartan in reducing mean 24-hour ambulatory DBP and SBP after 8 weeks of treatment (Oparil et al., 2001). Candesartan and irbesartan have potential uricosuric effects. Upon studies looked at the uricosuric effects of candesartan and irbesartan, no significant benefit with use of these agents was found (Wolff et al., 2015).

Table 2. Preferred ACEI, in terms of total respondents (total respondents $=60$ ).

\begin{tabular}{ccc}
\hline ACEI & No. of Responses & Percent of Total Respondents \\
\hline Captopril & 2 & 3.33 \\
Ramipril & 53 & 88.33 \\
Enalapril & 1 & 1.67 \\
Fosinopril & 0 & 0.00 \\
Lisinopril & 0 & 0.00 \\
Perindopril & 4 & 6.67 \\
\hline
\end{tabular}

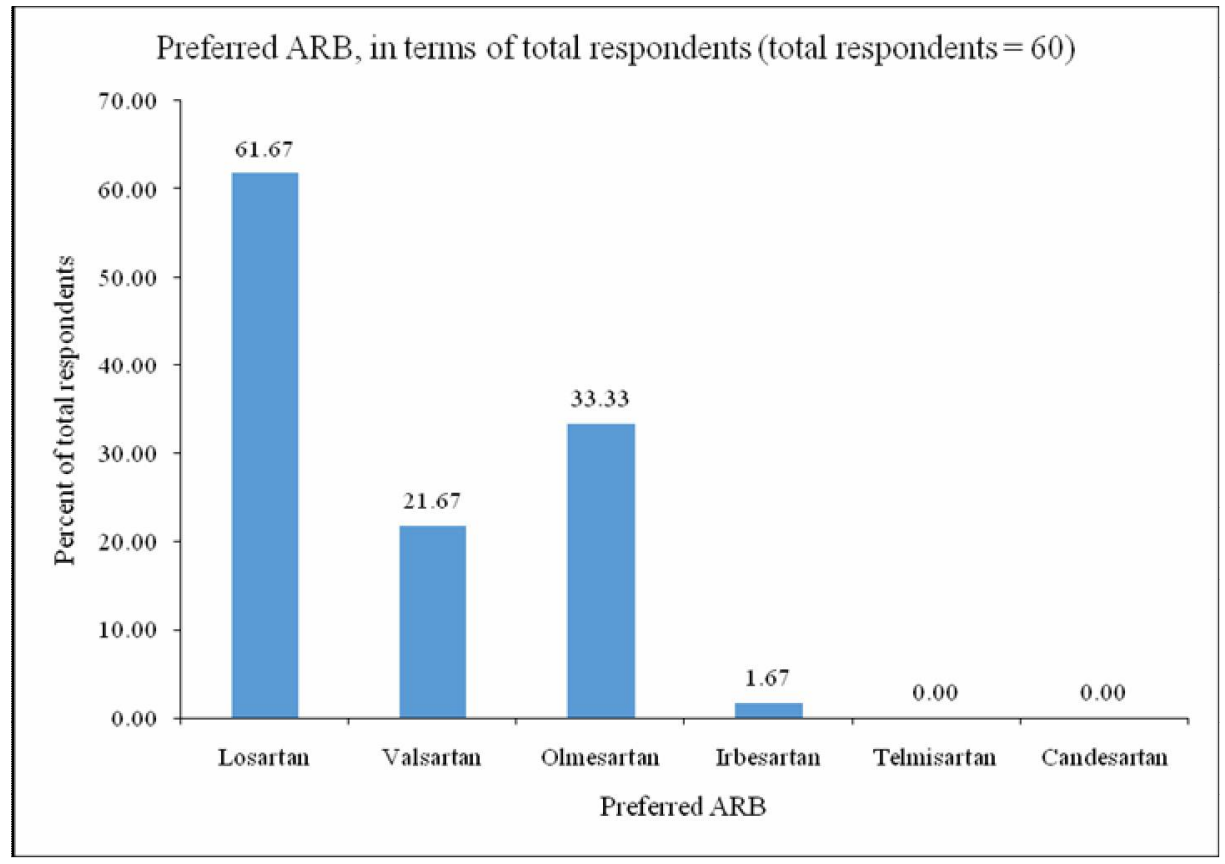

Figure 5. Preferred Angiotensin-II receptor antagonists (ARB).

Preferred CCB: $100 \%$ of the respondents agreed that they prescribed amlodipine most among the CCBs. However, few responded in favor of diltiazem (5.08\%). Other CCBs like nifedipine, lercanidipine, nimodipine, lacidipine and verapamil got no response. The reason why everyone opted for amlodipine was probably due to its longer duration of action than other CCBs like nifedipine. Amlodipine given once daily is at least as effective as nifedipine tablets given twice daily in patients with essential hypertension (Cappuccio et al., 1993). 
Preferred BB: It was observed that most of the respondents prefered bisoprolol (64.41\%) and metoprolol (38.98\%). But carvedilol (15.25\%), atenolol (1.69\%) and propranolol (1.69\%) got lower responses (Table 3). Labetalol and sotalol gained no response. The high preference of bisoprolol may be due to its advantage that it has a smaller impact on pressure and heart rate during the night (Soucek et al., 1993).
Preferred combination: Multiple answers were obtained in most cases to the question about preference of combinations. The most preferred (65\%) combination was amlodipine plus atenolol. On the other hand, $40 \%$ of the respondents prefered hydrochlorothiazide plus losartan and $33.33 \%$ prefer amlodipine plus valsartan. The minimum (8.33\%) was for indapamide plus perindopril.

Table 3. Preferred BB, in terms of total respondents (total respondents $=60$ ).

\begin{tabular}{lcc}
\hline BB & No. of Response & Percent of Total Respondents \\
\hline Metoprolol & 16 & 26.67 \\
Bisoprolol & 33 & 55.00 \\
Atenolol & 1 & 1.67 \\
Propranolol & 1 & 1.67 \\
Carvedilol & 9 & 15.00 \\
Labetalol & 0 & 0.00 \\
Sotalol & 0 & 0.00 \\
Others & 0 & 0.00 \\
\hline
\end{tabular}

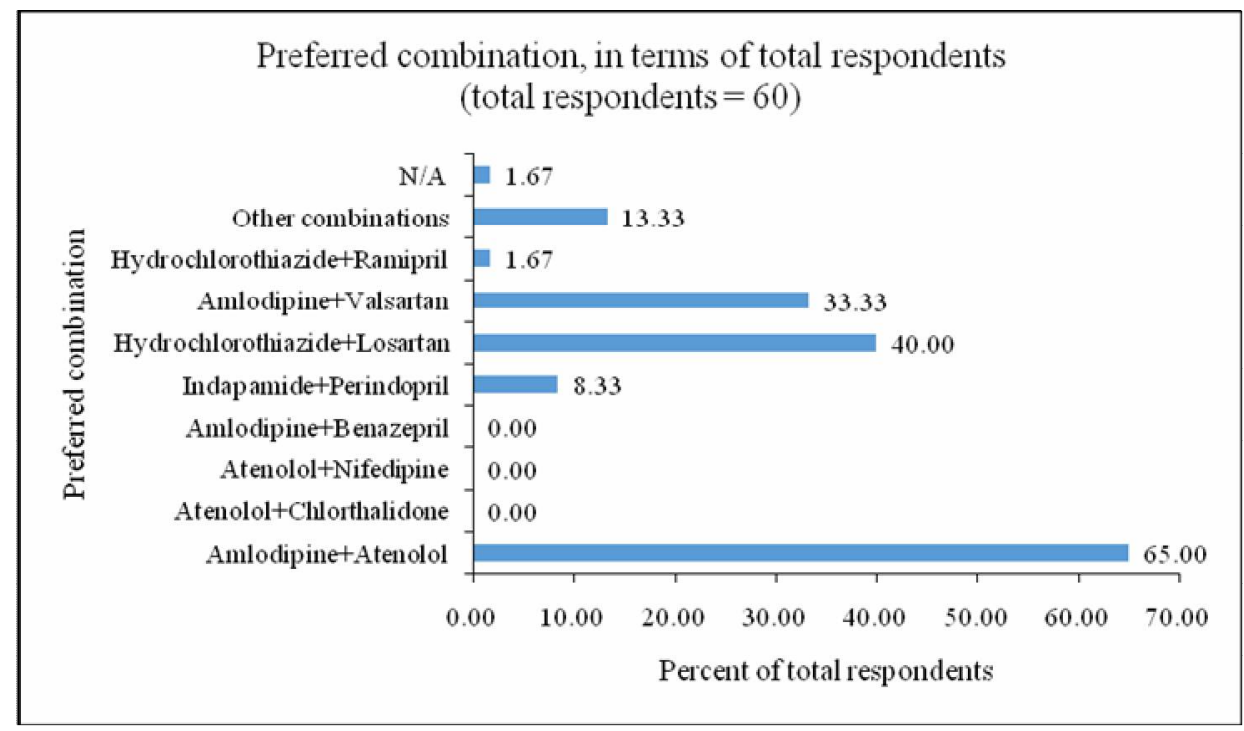

Figure 6. Preferred combination products in terms of total respondents (total respondents $=60$ ).

\section{Conclusion}

From this study, it has been appeared that the most renowned cardiac physicians of Dhaka city are keeping pace with the world practice. The limitation of the study was sample size and the findings obtained from this small sample size cannot be generalized to whole population of Bangladesh. However, a larger study involving physicians of rural areas can provide more precise data which will depict more clear scenario of cardiovasular drug-prescribing 
pattern. In addition, newer drugs like azilsartan (ARB), cilnidipine (CCB) etc. are now available in the market. Therefore, this work will help to guide to study what the physicians are thinking about newer drugs and more scopes for such comparative study in future.

\section{References}

Andrew, P.S. and Eugene, B. 2006. In: Harrison's Principle of Internal Medicine (Kasper D.L., Braunwald, E., Fauci, A.S., Hauser, S.L., Longo, D.L., Jameson, J.L., Eds.), McGraw-Hill Medical Publishing Division, 16e, Chapter 226 1434-1444.

Badiuzzaman, M., Mohammed, F.R., Chowdhury, F.R., Bari, M.S., Alam, M.B. and Ahasan, H.N. 2009. Prevalence of modifiable risk factors among stroke patients in a tertiary care hospital in Dhaka. $J$. Medicine. 10, 18-21.

Business Insights. 2011. In: The Cardiovascular Market Outlook to 2016. Business Insights Ltd., pp. 13-14, 46.

Cappuccio, F.P., Markandu, N.D., Singer, D.R., Crane, M., Carney, C. and MacGregor, G.A. 1993. Double-blind comparison between nifedipine and amlodipine for the treatment of essential hypertension. J. Hum. Hypertens. 7, 365-368.

Comeau, D.G., Sketris, I., Kephart, G.C., Bata, I.R. and Wolf, H.K. 1998. The change in composition and cost of antihypertensive drug treatment between 1985 and 1995 in the Halifax County MONICA area. Circulation. 97, 826-834.

Doggrell, S.A. 2001. Is Ramipril the pril for diabetes and kidney disease? Drugs Today (Barc). 37, 321-331.

Dukes, M.N.G. and World Health Organization. 1993. Drug Utilization Studies: Methods and Uses (Dukes, M.N.G., Ed.), WHO Regional Publications, European Series, No. 45, Introduction, pp. 1-4.

Gill, P., Stewart, K., Treasure, E. and Chadwick, B. 2008. Methods of data collection in qualitative research: interviews and focus groups. Br. Dent. J .204, 291295.

Hossain, M.S., Uddin, N., Islam AFM., Akash, R. H., Hasan, M. M., Saha, T. K., Hossain, M.M., Rahman, M.S. and Rana, M. S. 2015. Survey of hypertension in Dhaka, Bangladesh; changing prescribing patterns. Trop. J. Pharm. Res. 14, 329-335.
IMS Health, Bangladesh. 2016. IMS Health Bangladesh Ltd.

Kumar, M., Dahiya, V., Mishra, S., Sharma, D., Mishra, N. and Lahkar, M. 2016. Cardiovascular disease prevalence and drug utilization patterns at a tertiary care hospital in northeastern India. Int. J. Pharm. Pharm. Sci. 8, 116-119.

Labu, Z.K., Sultana, R., Bake, A., Sikder, K. and Jahan, K. 2013. Surveillance on prescribed cardiovascular drugs by generic names in Dhaka city of Bangladesh. Int. J. Pharm. Life Sci. 4, 2521-2520.

Lozano, R., Naghavi, M., Foreman, K., Lim, S., Shibuya, K., et al. 2012. Global and regional mortality from 235 causes of death for 20 age groups in 1990 and 2010: a systematic analysis for the Global Burden of Disease Study 2010. Lancet. 380, 2095-2128.

Manjula Devi, A.S., Sriram, S., Rajalingam, B., Alfet Raju, A., Varghese, R.S. and VenkataPhani, A. 2012. Evaluation of the rationality of fixed dose combinations of cardiovascular drugs in a multispecialty tertiary care hospital in Coimbatore, Tamilnadu, India. Hygeia J. D. Med. 4, 51-58.

Muhit, M.A., Rahman, M.O., Raihan, S.Z., Asaduzzaman, M., Akbar, M.A., Sharmin, N. and Faroque, A.B.M. 2012. Cardiovascular disease prevalence and prescription patterns at a tertiary level hospital in Bangladesh.J. Appl. Pharm. Sci. 2, 80-84.

Oparil, S., Williams, D., Chrysant, S.G., Marbury, T.C. and Neutel, J. 2001. Comparative efficacy of olmesartan, losartan, valsartan, and irbesartan in the control of essential hypertension. J. Clin. Hypertens. 3, 283-318.

Palinkas, L.A., Horwitz, S.M., Green, C.A., Wisdom, J.P., Duan, N. and Hoagwood, K. 2015. Purposeful sampling for qualitative data collection and analysis in mixed method implementation research. Adm. Policy Ment. Health. 42, 533-544.

Pendhari, S.R., Chaudhari, D.R., Burute, S.R. and Bite, B.M. 2013. A study on the drug utilization trends in the cardiovascular emergencies in a tertiary care hospital. J. Clin. Diagn. Res. 7, 666-670.

Soucek, M., Prásek, J. and Spinarová, L. 1993. Atenolol and bisoprolol in the treatment of mild and moderately severe hypertension. Vnitr. Lek. 39, 541-548.

Wolff, M.L., Cruz, J.L., Vanderman, A.J. and Brown, J.N. 2015. The effect of angiotensin II receptor blockers on hyperuricemia. Ther. Adv. Chronic Dis. 6, 339-346. 